\title{
El impacto de las prácticas neo-gerencialistas en las trayectorias científicas de hombres y mujeres en tiempos de crisis
}

\author{
Ana M. GONZÁLEZ RAMOS \\ Ester CONESA CARPINTERO \\ Universitat Oberta de Catalunya, Interdisciplinary Internet Institute (IN3) \\ agonzalezram@uoc.edu \\ econesaca@uoc.edu \\ Agnès VAYREDA i DURAN \\ Universitat Oberta de Catalunya, Estudis d'Arts i Humanitats \\ avayreda@uoc.edu
}

Recibido: Junio 2016

Aceptado: Noviembre 2016

\section{RESUMEN}

La incorporación paulatina de prácticas neoliberales en las organizaciones científicas ha dado lugar a profundos cambios en los modelos de carrera científica de hombres y mujeres. Actualmente, sus trayectorias se caracterizan por una intensa movilidad internacional y un modelo de progresión individualizada, puesto que las carreras profesionales no son sostenidas en una sola institución. La excelencia científica es el marco justificativo, ya que sólo aquellas personas que consigan un mayor número de méritos individuales podrán alcanzar las categorías superiores de la carrera profesional. Las políticas de austeridad imponen una constricción adicional, ya que los recursos para financiar nuevas incorporaciones e impulsar los procesos de promoción del personal de investigación se han reducido significativamente. Este trabajo se centra en las estrategias utilizadas por hombres y mujeres procedentes de distintas instituciones científicas donde operan diversos factores contextuales como las culturas científicas, el impacto de la crisis, etc. Los resultados muestran una variedad de situaciones sociales de hombres y mujeres en las instituciones científicas que, sin embargo, tratan de enfrentar las metas y prácticas que definen la excelencia científica.

Palabras clave: Políticas neo-gerencialistas, capitalismo académico, carreras sin fronteras, sesgos de género. 


\title{
The impact of the new managerial practices in the scientific trajectories of men and women in times of crisis
}

\begin{abstract}
The progressive incorporation of neoliberal practices in scientific organizations has provoked deep changes in the models of scientific career of men and women. Currently, their trajectories are characterized by intense international mobility and an individualized model of progression because professional careers are no longer sustained by one unique institution. Scientific excellence is the justificatory framework, since only those who reach a major number of individual merits will achieve the highest positions on the professional ladder. Austerity policies impose one additional constriction since resources for new enrollments and promotions have significantly decreased. This work focuses on the strategies used by men and women from different scientific institutions where various contextual factors operate, such as scientific culture, impact of the crisis, etc. The findings display a variety of social situations of men and women in scientific institutions that, however, try to deal with goals and practices that define scientific excellence.
\end{abstract}

Keywords: New Managerial Policies, Academic Capitalism, Boundaryless Careers, Gender Biases.

\section{INTRODUCCIÓN}

El concepto de "carrera científica" delimita las etapas que conforman la trayectoria profesional en la investigación. Las escalas laborales, descritas en los cuerpos legislativos y los convenios de trabajo, establecen un recorrido y qué criterios (méritos) hay que tener para acceder a categorías profesionales superiores (LOMLOU, 2007; Ley de la Ciencia, 2011). La descripción de estas escalas laborales proporciona un marco de referencia, al cual subyace cierta declaración de derechos (relacionada con el desarrollo profesional) y compromisos por parte de las organizaciones de sostener las carreras de investigación de su personal. La promoción es un derecho laboral que conlleva la estabilidad laboral, la mejora de las condiciones de trabajo y el salario, así como el reconocimiento de los logros profesionales. La crisis económica, junto a la implementación del neoliberalismo académico, han afectado profundamente al sistema de promoción en las universidades y centros de investigación.

Por una parte, la necesidad de racionalizar los recursos económicos debido a la situación de crisis ha paralizado los concursos del funcionariado y del personal laboral en las universidades. Las necesidades docentes se han cubierto con contratos de asociados de menor coste económico y que están desvinculados normativamente del derecho a la promoción profesional (la legislación dice que han de ser profesionales que aportan su experticia sobre la materia impartida y no se define ninguna carrera docente para este colectivo). También los centros de investigación han sufrido incidencias económicas que han afectado al desarrollo de las carreras científicas. Los grupos de investigación han recibido un número de proyectos inferior al esperado y de menor cuantía económica, por lo que cuentan con menos financiación para sostener sus equipos y personal de investigación y laboratorio. En algunos centros se ha reducido su 
plantilla por cuestiones de ahorro y viabilidad ${ }^{1} \mathrm{y}$, en general, se tiende a adoptar un sistema de contratación temporal. El número de categorías profesionales estables es excepcional en algunos centros altamente competitivos.

Las trayectorias científicas están afectadas por dos dinámicas reguladoras. Por una parte, la extensión de las políticas de gestión del personal neo-gerencialistas que provienen del liberalismo económico y, por otra parte, la implementación de un discurso de excelencia centrado en la internacionalización de la producción científica. El neo-gerencialismo (Deem, 1998, 2001) pone énfasis en la racionalización de los recursos y la maximización de los beneficios, por lo que desarrolla una fuerte orientación hacia la "marketización" de la producción científica y de la docencia (Slaughter y Rhoades, 2000, 2004). Por su parte, el discurso de la excelencia ha evolucionado hacia una mayor exigencia, contabilización de los méritos y selección de los criterios basados en la internacionalización. Los sistemas de evaluación de la calidad se han extendido y multiplicado generando cada vez mayor carga de trabajo y una gran presión en la cumplimentación de los requisitos (Morley, 2005; Mountz et al., 2015). Y el sistema de promoción descansa sobre patrones de evaluación considerados objetivos, aunque la subjetividad y la pertenencia a redes de influencia influyen inevitablemente en la toma de decisiones colectiva (Van den Brink y Benschop, 2012; Scully, 2002).

El ideal de excelencia científica tiene un impacto diferente en las carreras de hombres y mujeres puesto que un científico varón no verá interrumpida su carrera profesional por razones de maternidad (Bagilhole y Goode, 2001, González et al., 2015). En cambio, se ignoran los sesgos de género que se producen en las prácticas cotidianas, en los procesos de discriminación en los procesos de evaluación y estereotipos de género. Por tanto, como veremos a lo largo de este trabajo, las mujeres siguen trayectorias similares a los hombres en el sentido de que tratan de alcanzar los mismos hitos a pesar de enfrentarse a situaciones sociales (tanto en la familia como en las organizaciones científicas) diferentes a las de los hombres. Sus carreras se ven, en mayor medida, afectadas por el abandono, un ritmo más lento o una menor valoración de sus méritos profesionales.

El objetivo de este trabajo es analizar las estrategias de hombres y mujeres procedentes de distintas instituciones científicas donde operan diversos factores contextuales (cultura científica, impacto de la crisis, etc.) en el contexto de crisis económica y de adopción de un modelo de gestión neo-gerencialista. Para aproximarnos a este fenómeno, hemos utilizado una metodología cualitativa basada en diez estudios de caso llevados a cabo en diferentes centros de investigación y departamentos de universidades del estado español. El trabajo se divide en cuatro apartados. La primera sección presenta algunas aportaciones teóricas que permiten interpretar los discursos del personal de investigación. La segunda sección presenta las características de los centros incluidos en este estudio y la metodología de

1 Por ejemplo, "El CNIO acuerda reducir su plantilla con 31 bajas voluntarias y ningún despido" según el titular de rtve de 08.01.2014, https://goo.gl/UdPAMh 
acercamiento al objeto de estudio. La tercera expone las narraciones de estas personas a propósito de sus carreras científicas en su contexto institucional. Por último, la cuarta sección, sintetiza los resultados sobre los modelos de carrera científica implementados en las instituciones científicas.

\section{VARIACIONES SOBRE UN TEMA, LA(S) TRAYECTORIA(S) CIENTÍFICA(S)}

La definición de una trayectoria profesional transparente refleja una política de gestión de recursos humanos eficiente y responsable. La Carta Europea para los Investigadores (European Commission, 2005: 18-19) recoge la necesidad de establecer una carrera investigadora que esté prevista en las unidades de gestión de recursos humanos, y guiada por mentores que tienen la misión de motivar a los investigadores, en cualquier etapa en la que se encuentren, con el fin de reducir la incertidumbre sobre su futuro profesional. Actualmente en España contamos con diversas normativas aplicables a diversas instituciones (universidades, CSIC y centros de investigación regulados por varios organismos), tipos de profesionales (por ejemplo, funcionarios y laborales), vinculadas a las propias organizaciones (normas internas y convenios laborales, dependiendo de la naturaleza jurídica del centro) y por agencias de evaluación externas (que certifican la independencia de la acreditación de méritos). Como resultado de todo ello, podemos afirmar que los modelos de carreras científicas presentan diversas variaciones sobre un mismo tema.

Igualmente, las diferentes culturas científicas han definido históricamente el modelo de ciencia predominante: qué criterios son reconocidos, cómo se desarrollan las trayectorias de excelencia, qué tipo de movilidad se deben realizar. Más allá de las de estos criterios generales, cada área define una cultura propia que influye en las carreras profesionales del personal de investigación. Pongamos el caso de la astrofísica y la arqueología. Ambas disciplinas necesitan realizar trabajo de campo en localizaciones específicas (la astrofísica en observatorios, la antropología en los yacimientos históricos). Mientras la astrofísica ha desarrollado una movilidad constante entre observatorios, la arqueología ha adoptado una estrategia de estancias periódicas en los diversos campos de trabajo.

Además, la movilidad se ha extendido a otros campos del conocimiento como consecuencia de la alta especialización tecnológica (puesto que las tecnologías más avanzadas están localizadas en ciertos laboratorios e instalaciones científicas) y la extensión de una cultura de la excelencia basada en la internacionalización (ya que las mejores investigaciones se producen en un número limitado de institutos $o$ universidades). Cuando la cultura científica gira alrededor de una red de institutos de investigación que trabajan sobre un campo del conocimiento específico (por ejemplo, la robótica) el nomadismo científico se ha establecido como pieza fundamental de creación del conocimiento (Bozeman et al., 2013).

El concepto de "carreras sin fronteras" (boundaryless careers) fue propuesto por Arthur y Rosseau (1996) para constatar la nueva forma de relación contractual establecida entre el personal altamente cualificado y las organizaciones científicas. En 
dicho modelo prevalece la "agencia" de las personas que investigan, quienes planifican y gestionan sus propios recursos para desarrollar una carrera exitosa, es decir, que se responsabilizan de los resultados logrados en su carrera de investigación (Arthur y Rosseau, 1996; Sullivan y Arthur, 2006). Los investigadores "eligen" su trayectoria laboral mediante la consecución de contratos y becas desempeñadas en diferentes organizaciones que en gran medida, se localizan en distintos países. Así, las organizaciones científicas quedan liberadas de la responsabilidad de ofrecer posiciones de promoción a sus trabajadores a lo largo de su carrera.

Según algunos autores (Roper et al., 2010: 673, Clarke y Knights, 2015), esta dinámica es una extensión de la política económica neoliberal que enfatiza el individualismo frente a la lógica corporativa. En contraposición a los derechos laborales que han sido conquistados por los trabajadores de manera colectiva en las organizaciones, la gestión de recursos humanos neo-gerencialistas se basa en la gestión autónoma de las carreras que consiguen diferentes compromisos y salarios dependiendo de la negociación individual con los gerentes. De esta manera, las instituciones consiguen racionalizar los costes del personal y la fidelización de la élite de trabajadores respecto a los cuales adquieren la responsabilidad de proporcionar unos derechos laborales vitalicios. Ello conlleva una gran presión sobre las personas trabajadoras, que se ven obligadas a demostrar continuamente su productividad (Trow y Clark, 1994; Fassa, 2013).

La extensión de las agencias de evaluación de la calidad (Morley, 2005; Baker, 2010; Mountz et al., 2015) forma parte de este entramado siguiendo la lógica de la contabilización de méritos considerados excelentes. Las instituciones científicas consiguen mejorar sus indicadores gracias al paso de investigadores e investigadoras altamente competitivos que dejan un rastro significativo en las instituciones de acogida en forma de publicaciones, patentes, proyectos financiados y becas conseguidas, pero que han de marcharse cuando finaliza su beca o proyecto. La fuerte competitividad y la falta de posiciones provoca estrategias personales individuales que sustituyen a las estrategias colectivas para enfrentar la precariedad (Clarke and Knights, 2015). Como Morley (2016) apunta, "la academia neoliberal, aunque no esencialmente masculina, puede reforzar masculinidades particulares, produciendo una cultura de la virilidad, que valora las personas en relación a la cantidad de dinero que consiguen - el homo economicus" (Morley, 2016: 32).

De acuerdo con Inkson et al. (2012), las dinámicas globales tensionan las carreras cosmopolitas respecto a las carreras locales. La fuerte vinculación entre la internacionalización y la excelencia devalúa aquellas carreras menos internacionales y centradas en la creación de conocimientos locales. Los centros de investigación y las universidades están obligados a internacionalizar sus resultados para aumentar su competitividad y su posición en los rankings (Baker, 2010), por lo que, aquellas instituciones más orientadas a referentes locales y con menor orientación internacional parecen abocadas a producir resultados menos "excelentes".

En los centros con intensa movilidad internacional no hay apenas personal en posiciones estables (sino contratos cuya duración no supera los cinco años); y las personas que investigan desarrollan su trayectoria optando a becas y contratos en 
alguno de los centros que forman parte de la red de "centros excelentes". Por el contrario, en algunas disciplinas y universidades, la movilidad es temporal y, por tanto, el personal académico retorna a la misma institución (en ocasiones, la movilidad incluso es patrocinada como parte de la estrategia de formación y promoción de su personal de investigación). Del mismo modo, las empresas tecnológicas, a diferencia de las multinacionales, valoran en menor medida las carreras internacionales en contraposición a "la cantera" (es decir, el talento formado en el ámbito local y cuyo trabajo repercutirá en la misma región). Por su parte, las empresas potencian las patentes y la transferencia de tecnología por encima de las publicaciones puesto que su orientación es el mercado y la creación de valor monetario. Los proyectos exitosos permiten evidenciar la valía de los profesionales. Mientras tanto, la jerarquía es mucho más vertical que en los centros de investigación y universidades. En resumen, empresas, universidades y centros de investigación crean diferentes prácticas y discursos sobre la promoción y la captación de talento, que responden a su propia adaptación neo-gerencialista de gestión del personal de investigación.

Además de estas variaciones que provienen del ámbito institucional, hay que añadir la compleja relación entre trabajo y vida personal que influye en las decisiones tomadas por hombres y mujeres investigadores (Brocklehurst, 2003; Ackers, 2004; González y Torrado, 2014). Los hijos y las parejas, la necesidad de reforzar las redes familiares tras un largo período en el extranjero, y la enfermedad o los cuidados de un familiar suelen ser motivos esgrimidos tanto por hombres como por mujeres para retornar (Yeoh y Khoo, 1998). Sin embargo, las mujeres están vinculadas con esas tareas sociales más fuertemente que los hombres. Los hombres que lo hacen son recompensados mientras para las mujeres es una obligación de la cual se derivan consecuencias sociales y emocionales.

La categoría de género está atravesada por factores de clase, origen geográfico y etnicidad, las cuales influyen de manera específica en las trayectorias de investigación. La posición de clase, origen étnico y geográfico repercute tanto en el acceso a los recursos como en la planificación de la carrera y en la estrategia familiar. Ello influye en las metas a perseguir por las mujeres y en los resultados logrados profesionalmente. Hombres y mujeres de distinto origen social desarrollan diferentes estrategias que, en primer lugar, impactan sobre sus trayectorias profesionales y, en segundo lugar, sobre las representaciones sociales que construimos acerca de ellos y ellas. Estas expectativas sobre su capacidad influyen en las metas profesionales que lograrán. Por ejemplo, esperamos que un hombre indio sea bueno en matemáticas pero no esperamos que una mujer iraní ganase la medalla Fields.

Sobre este tapiz lleno de posibilidades, las personas trazan distintas trayectorias de investigación. La objetividad y la transparencia de los procesos de evaluación -que se identifican con la excelencia científica- refuerzan el esfuerzo realizado por los jóvenes para avanzar a lo largo de la trayectoria laboral (Rees, 2011). A pesar de ello, los sesgos producidos por expectativas estereotipadas de género, clase y etnia producen desviaciones respecto a los principios de identificación del talento (Bagilhole y Goode, 2001; Van der Brink y Benschop, 2012; Scully, 2002). Además, la crisis económica justifica la modificación de las normas que regulaban la progresión profesional, puesto 
que, aun cumpliendo los requisitos meritocráticos, la estabilidad laboral y la promoción profesional no están aseguradas. Uno de los efectos de la crisis ha sido la "cola" de candidatos y candidatas con acreditaciones y cumplimiento de méritos suficientes, que se están viendo obligadas a prolongar el tiempo de espera hasta lograr una posición superior o que, en el mejor de los casos, se han visto obligados a encontrar becas en otros países (la llamada fuga de cerebros). En el peor de los casos, algunas personas desistirán de continuar una trayectoria inestable e incierta, abandonarán la investigación, y aceptarán una posición técnica, de gestión de proyectos $\mathrm{o}$, simplemente, cambiarán completamente de profesión. Estas personas, como veremos en los resultados de este trabajo, son mujeres en mayor medida (a veces, parejas de un compañero científico que, sin embargo, continúa progresando académicamente).

\section{METODOLOGÍA DE INVESTIGACIÓN}

El presente trabajo está basado en los resultados de diez estudios de casos realizados dentro del marco de un proyecto más amplio, GENERA: Generación de una economía del conocimiento más inclusiva y competitiva (FEM2013-48225-C3-1-R), que tiene como objetivo identificar los sesgos de género en las instituciones científicas. Con el objetivo de identificar las estrategias de hombres y mujeres en distintas instituciones científicas y qué modelos de trayectorias adoptan de acuerdo a su cultura, políticas neo-gerencialistas e impacto de la crisis económica se ha llevado a cabo diez estudios de casos. En concreto, se eligieron dos instituciones del campo de las tecnologías (informática y telecomunicaciones), dos de ecología (marina y forestal), dos de ciencias de la salud (biomedicina y biología molecular), una de ciencias experimentales, una de humanidades, una de ciencias sociales y una de arquitectura. Cuatro de esas instituciones pertenecían al ámbito académico universitario, cuatro a centros de investigación con financiación mixta, una al CSIC y una empresa de innovación, situadas respectivamente en Cataluña, Madrid, Andalucía, País Vasco y Galicia respectivamente.

La cultura institucional de estos centros demuestra una gran variabilidad respecto a su productividad, internacionalización y competitividad lo que influye en las prácticas de gestión del personal de investigación y en la autogestión de las carreras científicas. Así pues, las unidades de observación presentan perfiles muy variados que podemos situar en algún punto concreto de entre estos tres tipos de centros:

- Centros de investigación (con financiación privada y pública) altamente competitivos y orientados a la internacionalización de su investigación, con un porcentaje de posiciones estables muy reducido, y un bajo nivel de compromiso en la promoción de las carreras de investigación de su personal.

- Departamentos de universidad, desde algunos altamente competitivos y claramente orientados a la investigación hasta otros más orientados a la docencia de grado y no a la investigación; con un elevado número de funcionarios y posiciones fijas o con un alto número de 'falsos asociados' y, en general, de investigadores contratados en condiciones precarias. 
- Empresa de innovación con una fuerte orientación hacia la investigación nacional requerida por el mercado local (puesto que no pertenecía al grupo de empresas multinacionales) y con una fuerte participación en consorcios internacionales para llevar a cabo proyectos de investigación. El personal de investigación trabaja con ritmo empresarial, ejecutando proyectos solicitados por los clientes a los que tiene que "vender el producto". Los equipos de trabajo son jerarquizados y su plantilla es mayoritariamente local que, en cambio, cuenta con bastante probabilidad de estabilidad laboral.

La aproximación metodológica a las unidades de observación fue la misma para todos los casos de estudio. Una vez realizado el contacto y adquiridos los compromisos de colaboración entre la institución y el equipo de investigación, se emprendió una fase de investigación sobre la documentación institucional. El análisis se centró en la normativa y los materiales informativos sobre el centro, los medios de difusión on-line y off-line de cada centro (página web, trípticos, dosieres, etc.).

En segundo lugar, se realizaron entrevistas personales para conocer las trayectorias de los hombres y las mujeres investigadoras. El número de entrevistas era variable en cada centro dependiendo de la dimensión de la unidad elegida (la unidad esencial en la universidad era el departamento y el centro de investigación en el resto de los casos, por lo que el número de personas integrantes de cada unidad era diferente). En cada centro se eligieron un hombre y una mujer de cada categoría laboral que representaban posiciones significativas de la carrera profesional en la institución (por ejemplo, líder de grupo, staff scientists, postdoc; catedrático, titular de universidad, contratado doctor y ayudante doctor). Por tanto, contamos con el relato de personas situadas en diferentes momentos de su trayectoria científica. En las entrevistas, se exploró tanto el pasado, como el presente y las expectativas futuras. En total, se realizaron alrededor de 80 entrevistas de una duración de una hora a dos horas, completamente balanceadas en términos de género.

En tercer lugar, la persona encargada de cada estudio de casos elaboró una representación gráfica de la carrera profesional de su informante (egogramas) y lo retornó a cada persona de manera que pudiera validar la información contenida en ella. De este modo, también se validó la interpretación que la entrevistadora sobre los discursos de las personas entrevistadas. En los egogramas se señalaron los principales hitos personales y profesionales, reflejando las interrupciones o continuidades en la trayectoria laboral, y las personas o elementos que facilitaron o dificultaron su trayectoria profesional y su vida personal. Una ventaja de esta metodología es que permite validar la interpretación que la entrevistadora hace sobre las biografías de las personas entrevistadas. La limitación de esta metodología se ciñe a la dificultad de sistematizar la información de manera analítica. En cambio, supone un documento de trabajo adicional de debate y de reflexión sobre la estrategia descrita por cada informante en un momento determinado (durante el proceso de implementación de la entrevista) que se extenderá en un momento posterior.

En cuarto lugar, se realizó una reunión colectiva con investigadores senior que usualmente son elegidos como miembros de los comités de selección y contratación del centro. Dependiendo del tamaño de la unidad del centro, los comités están formados 
entre 3-5 personas, siempre prefiriendo número impar de componentes total y con la mayor diversidad de género (por ejemplo, dos mujeres y hombre o tres mujeres y dos hombres). En dicha entrevista se simuló un proceso de selección entre dos candidatos imaginarios, lo más parecido posible a la dinámica utilizada en cada centro para incorporar personal doctor. Para ello, se les mostraba dos currículos, de un hombre y de una mujer, con características diferentes (se introducía un elemento de variación dependiendo de la disciplina y de los factores que se juzgaban más significativos para cada centro). Así, por ejemplo, se introdujeron diferentes factores, como que fueran candidatos internos o externos, que estuvieran más orientados a la docencia o a la investigación, que presentaran cartas de recomendación más o menos extensas, pobres o detalladas en información personal, etc. Durante la reunión se invitó a los supuestos miembros del tribunal a discutir sobre sus perfiles y a consensuar un candidato. Una vez tomada la decisión, se daba por finalizada la reunión, y se les pedía que respondieran a un pequeño cuestionario (individual y anónimo) en el que se les preguntaba por el proceso de decisión (si estaban satisfechos con el acuerdo, si consideraban que el consenso había sido fácil o difícil, quién y con qué argumentos había influido en su voto final).

En último lugar, se elaboró un informe describiendo el clima de la unidad de estudio de casos, las principales características respecto al equilibrio de género y la igualdad de oportunidades. Este documento, además de responder a la necesidad de devolver la información a la población de estudio (Taylor y Bogdan, 1984), valida la interpretación realizada por parte de la persona encargada del estudio de caso, suscita nuevos comentarios de los participantes en el estudio y a una nueva reflexión sobre los discursos construidos por las personas involucradas y la investigadora.

La información obtenida a través de esta metodología es muy amplia y, por tanto, difícil de sistematizar en un sólo artículo. Así que, en este trabajo se enfatiza aquella información obtenida sobre el impacto de las prácticas neo-gerencialistas en cada una de las metodologías aplicadas y acerca de la excelencia en los procesos de incorporación y promoción. También se refleja aquellos rasgos emergentes debido a los efectos de la crisis que impregnan las políticas de (in)estabilización y las condiciones laborales del personal de investigación. Por esta misma complejidad de la información, se muestran las citas de las biografías y no de las interacciones posteriores o de las reuniones grupales que serán expresadas en trabajos posteriores.

\section{ESTRATEGIAS DE ÉXITO, ESTRATEGIAS DE RESISTENCIA}

Aunque el título de esta sección refleja dos situaciones antagónicas, las dos ideas sintetizan una realidad comúnmente experimentada por los investigadores e investigadoras a lo largo de sus trayectorias científicas de manera secuencial y continuada. Durante la carrera de investigación se producen diversos acontecimientos que terminan por dibujar su trayectoria profesional (González, 2014), factores externos que determinan sus oportunidades laborales (la creación de nuevas universidades y centros de investigación o la ausencia de recursos, el número y el nivel de los candidatos en competencia, el apoyo o la obstaculización de los colegas y supervisores, 
etc.) más allá de sus méritos y acreditaciones. Dichas variaciones también están ligadas a factores culturales (de organización de las instituciones y de las disciplinas de conocimiento) e individuales (desde rasgos de personalidad hasta condiciones personales y familiares). Incluso ante situaciones parecidas, hombres y mujeres abordan las decisiones de manera diferente según sus roles de género. En esta primera parte de los resultados, diversos ejemplos ilustran esta circunstancia.

Generalmente, las personas que se dedican a la investigación suelen mostrar una fuerte implicación en su trabajo científico, unas expectativas altas respecto a sus competencias, y una fuerte determinación respecto a sus metas profesionales (Fusulier, 2016). Dicha actitud les permite afrontar numerosas dificultades y decepciones a lo largo de su trayectoria científica. El sacrificio y la superación de las dificultades forman parte de las trayectorias de investigación tanto como los éxitos. Así relata su incorporación al mundo de la ciencia, un investigador senior en un centro mixto de investigación centrado en la ecología:

[Sobre la beca de doctorado] “...y el primer año no me la dieron. El segundo tampoco. El tercero tampoco. Y el cuarto año, sí. El último año que me la podían dar, me la dieron [risas]. Porque también tenía claro que, si podía, quería hacer investigación" [Hombre, 45 años, sin pareja ni hijos, director de proyectos, centro mixto].

Las decisiones sobre las relaciones personales no son independientes de las estrategias profesionales (González y Vergés, 2013). Los investigadores sienten estas dos circunstancias como factores entrelazados que impulsan o limitan sus carreras profesionales en momentos decisivos de su trayectoria profesional. Estas encrucijadas entre su vida profesional y personal están relacionadas con el cuidado, retos profesionales estimulantes, negociaciones con la pareja, preferencias por un país y estilo de vida determinados, entre otros factores. Además, como ya afirmamos en un trabajo anterior (González y Torrado, 2014) las razones aparecen entremezcladas en su discurso sin poder discernir la primacía de unas sobre otras.

Entrevistado [E]: "En la Universidad del Estado de Louisiana. Louisiana State University, que está en Baton Rouge. Y estuve allí, bueno, 15 días. Después de 15 días cogí y dije 'Este pueblo no me gusta... y me marcho' Y...

Investigadora [I]: Y ¿por qué no te gustaba?

E: Bueno, a ver, Louisiana es uno de los Estados de Estados Unidos más oprimidos. Es uno de los 3 Estados, junto con Mississippi y Alabama seguramente más... más... eh... más pobres, donde también han robado más, pero eso es históricamente, no viene al caso. Y... y bueno, luego también, en esa época tenía un problema fa... familiar, porque mi madre se había puesto enferma. Entonces, eh...justo... justo en aquella época le habían operado por tercera vez un cáncer y le tenían que volver a dar quimioterapia. Y...y bueno, en ese momento pues, en plan, sumé todas las variables, puse en la balanza las cosas que me importaban en ese momento y dije: "mira, yo prefiero marcharme de aquí... y... ¡y cuidar de mi madre!". También es cierto que... tomé la decisión... creo que fue en septiembre, cuando una tormenta tropical llegó a Baton Rouge y... yo estaba 
sin coche y... bueno, estuve como una hora caminando bajo el agua... tuve que andar en plan... tres millas, o a no sé cuántas, hasta donde estaba el departamento. Y dije: '¿Esto no es vida!'. O sea, simplemente... Entonces, fue cuando dije 'yo me voy de aquí'” [Hombre, 34 años, sin pareja ni hijos, programador, empresa tecnológica].

La pasión y los éxitos están acompañados por momentos de sacrificio (Vázquez y Elston, 2006), sobre todo, en el caso de las mujeres. Por lo que se refiere a la movilidad, la estabilidad emocional, la negociación con las parejas y el cuidado de las familias marcan profundamente la decisión de retornar al país de origen (Yeoh y Khoo, 1998; Xie 2010; González y Torrado, 2014). Debido al rol de género adjudicado socialmente, este tipo de decisiones se viven de manera aceptada y menos cuestionada (Baker, 2010). Incluso en casos como el siguiente en que la investigadora entrevistada recalca previamente el sentimiento de bienestar y buen ambiente científico en Alemania donde desarrollaba satisfactoriamente su carrera científica:

E: "Son muchos años en un país. Entonces pensé, ¿bueno nos volvemos o nos quedamos?

I: ¿y tu pareja había venido contigo?

E: No, nos conocimos allí en [centro prestigioso]. Él había llegado creo que un año antes que yo (...). Y luego pensamos, “¿qué hacemos?” porque... O sea hay un momento en el que tienes que... ¡Nos organizamos la vida! Entonces a mí me ofrecían en Berlín un trabajo, y en el mismo instituto también le buscaban a él opciones. Y después surgió esto de [centro prestigioso español] y bueno, a [él] no le gustaba mucho Berlín y me pareció que era mejor movernos. (...). Entonces nos vinimos, yo con una plaza de ICREA [programa de excelencia catalán] y [él] sacó... Primero tenía un Ramón y Cajal y luego sacó la plaza en el CSIC y ya nos organizamos aquí la vida. (...). Es que llega un momento en que empiezas a pertenecer al sitio donde vives. Y... A mí me gustaba, aún sigo yendo, sigo manteniendo mucho contacto con Alemania y me sigo sintiendo en casa. Pero claro nos tiene que gustar a los dos... Y bueno también, claro, yo ya tenía a mi madre que ya se iba haciendo mayor aquí en España y llevarla a Alemania tampoco era una cosa... no es un país... no es un idioma sencillo. Y bueno, al final decidimos que aquí.

I: Pero por lo que veo por ti te hubieras quedado, ¿no?

E: Sí, me hubiera quedado, sí (risas). Si no hubiera tenido que pensar en otras personas me hubiera quedado sin duda, sí." [Mujer, 52 años, con pareja, sin hijos, líder de grupo, centro mixto].

\section{TRAYECTORIAS CON REDES DE APOYO Y TRAYECTORIAS INDIVIDUALIZADAS}

Las carreras científicas son el resultado de un proceso, a veces consciente (vocación), a veces, inconsciente que se va construyendo poco a poco: "Fue la vida 
que me fue llevando por allí” [Mujer, 49, casada segundas nupcias sin hijos, ecología, centro mixto]. Están fuertemente ligadas a las oportunidades laborales, y a la obtención del apoyo necesario para conseguir una posición sólida: “...Y me llegó una oferta porque había trabajado con ellos dentro de la universidad, de una empresa conocida en... en Bilbao, y la acepté" [Mujer, 32 años, en pareja, sin hijos, programadora, empresa tecnológica].

“Entonces, yo entré guiada, pero yo me 'jarté de currar'. Entonces, se presentó la beca, yo solicité la beca y, nos la dieron a varios, no sólo a mí. Varios entramos y hicimos... había que diseñar un laboratorio, una red de un laboratorio... Pues, entre varios lo hicimos. Yo cobré mi beca y ya tenía mi mérito. Y después me dijo el director [del departamento], antes de que hubiera plazas libres: 'Mira, hay un... yo tengo un proyecto, tengo que contratar a alguien...' . Me lo ofreció a mí. Se lo podía haber ofrecido a otro, pero lo me ofreció a mí. Y me contrató tres meses para hacer un trabajo, y yo lo hice, cobré y ¡ya está! ¡Y ya tenía esos puntos! Cuando llegó la hora de que había plazas libres, cuando la gente se presentó, yo era la que tenía más puntos". [Mujer, 39 años, divorciada, un hijo, ayudante doctor, universidad].

Las carreras profesionales son más fluidas si se cuenta con personas que las apoyan, orientan a la persona candidata sobre los próximos pasos a dar o, simplemente, las incluye en proyectos del grupo. Las relaciones personales con profesionales sénior son en unas ocasiones más visibles que en otras en los discursos de las personas entrevistadas, curiosamente, tanto si han ejercido una influencia positiva como si han ejercido una influencia negativa en sus carreras profesionales. Es posible encontrar casos que han conseguido una posición permanente a pesar de carecer de apoyos personales en la institución. Estas personas han hecho valer los méritos alcanzados, necesariamente superiores a los del resto de personas candidatas, gracias a la aplicación de un baremo objetivo que les sitúa por encima del resto. Todo ello a pesar de que la situación de crisis no ofrece oportunidades de incorporación que puedan aprovechar las personas con los méritos necesarios (a veces muy superiores) para cubrir esas posiciones de promoción. Muchas veces incluso a pesar de tener un sistema de apoyos positivos y circunstancias favorables en sus centros de trabajo.

La mujer de la siguiente cita ha encontrado diversos problemas a lo largo de la carrera porque carecía de una red que la apoyara en su departamento:

"El director de mi tesis era el director del departamento, que no me había avisado que salía la plaza, aunque yo le había dicho que estaba interesada. Pero es que el que la había ocupado antes era su favorito. Y, entonces, que pasara yo a ocupar esa plaza... pues era muy duro. Por eso no me avisaron. Y cuando pasó todo eso, yo conseguí quedarme, y este hombre me dijo que no me seguía dirigiendo la tesis y tuve que cambiar de director de tesis" [Mujer 52 años, con pareja, sin hijos, arquitectura, titular de universidad].

Por tanto, ha realizado una carrera discontinua, ya que ha "abandonado" la carrera científica en diversas ocasiones, desviándose de la trayectoria lineal que supuestamente debía desarrollar un investigador excelente. Sin embargo, ella ha estado desvinculada 
varias veces de la universidad, tanto para realizar estancias duraderas en el extranjero como para ocupar puestos en instituciones no académicas. A pesar de la oposición de los grupos de interés en su departamento, ha alcanzado la plaza de titular, y sigue trabajando para lograr la posición de catedrática. En su caso podemos confirmar que, pese a la falta de apoyos, una evaluación objetiva de los méritos ha permitido una evaluación positiva. Sin embargo, la mayoría de los relatos de las personas entrevistadas dejan entrever que, incluso sin haber nepotismo en la asignación de plazas, el conocimiento previo de la persona candidata es una ventaja fundamental para ser evaluada positivamente e, incluso, contar con la información necesaria para participar en el concurso de méritos es una condición previa para promocionarse. Por otro lado, también se puede afirmar que conforme las carreras son más individualizadas, la capacidad de dirigir una carrera de investigación hacia una posición de cátedra o dirección en la universidad tiene menos probabilidad de éxito para esas personas. En algún punto de la carrera tienen que conseguir redes suficientemente influyentes para que apoyen su candidatura, hecho que está cargado de connotaciones de género.

Las "carreras sin fronteras" (Arthur y Rosseau, 1996; Sullivan y Arthur, 2006) son un ejemplo de carreras individualizadas pero debidas a una dinámica institucional. En tres de los cinco centros de investigación incluidos en este estudio, la mayoría de las figuras contractuales son temporales y las carreras de investigación se construyen a partir de la movilidad entre centros pertenecientes al mismo campo de especialidad. A través de estas movilidades sucesivas se transfiere el conocimiento entre los grupos de investigación, el candidato adquiere experiencia, acumula los méritos curriculares deseados y, finalmente, consigue autopromocionarse en las redes de influencia (Roper, 2010; Inkson, 2012). De este modo, los y las jóvenes doctores van sumando numerosas becas postdoctorales en diferentes países. El siguiente investigador es, justamente, un contraejemplo pero con su discurso nos muestra cuál es la "norma": "Soy un postdoc atípico porque he hecho una postdoc de nueve años en un sólo sitio. Esto no es típico" [Hombre 34 años, sin pareja ni hijos, postdoc, centro mixto].

En los centros red, donde incluso los directores y las directoras de los grupos de investigación desarrollan carreras nómadas, es fundamental contar con la confianza de los superiores para continuar la trayectoria de investigación. Si un o una líder de grupo se traslada a otro laboratorio, puede llevarse consigo a uno o más miembros de su equipo. Los miembros que no son "convocados" por el o la líder del grupo, no sólo pierden su contrato sino todo el trabajo de investigación que estuvieran desarrollando; puesto que el experimento no puede continuar sin el laboratorio, que desaparecerá con el líder del grupo, esta persona se quedará sin su línea de investigación.

La crisis económica y la menor aportación financiera conseguida a través de los proyectos ha perjudicado la incorporación de un mayor número de personas en los últimos años. Las trayectorias de las mujeres se resienten en mayor medida por esta circunstancia, puesto que los y las líderes de grupos (la mayoría hombres) no suelen elegirlas ni siquiera valorarlas como investigadoras potentes: "yo no he oído nunca, nunca jamás en este centro que a mí se me alabe por mis dotes de investigadora, en cambio sí que se valora mucho mis dotes de gestión" [Mujer, 47 años, con pareja, sin 
hijos, ecología, centro mixto]. Como se afirma en esta cita, las trayectorias de otras mujeres también confirman la influencia de las decisiones de sus jefes y las expectativas que tienen sobre ellas. Son ellos quienes les han encargado de la organización del laboratorio, la puesta en marcha de las aulas de prácticas o el desempeño de tareas burocráticas en lugar de apoyarlas para que tomen responsabilidades relacionadas con la investigación. Las mujeres no suelen otorgar a esta situación una categoría de discriminación, puesto que consideran que es una situación circunstancial asociada únicamente a ellas mismas (Kelan, 2009); sin embargo, la repetición de esta pauta de comportamiento permite constatar que no es una práctica aislada sino un sesgo de género.

\section{NEO-GERENCIALISMO Y EXCELENCIA EN TIEMPOS DE PRECARIEDAD}

El neo-gerencialismo ha modificado la relación contractual del personal investigador con las instituciones científicas. Sirva como ejemplo el caso de un centro mixto, con fuerte financiación del gobierno autonómico, donde la falta de financiación ha cambiado su modelo de gestión económica:

"Lo que pasa que ha ocurrido... lo que ha ocurrido, es que ha habido recortes y... ya... ahora lo que nos ha ocurrido a todos los investigadores de plantilla... es que una parte de nuestro sueldo, de alguna manera, depende de proyectos; que hasta entonces, más o menos [la partida de personal] quedaba cubierta simplemente sin...Teníamos proyectos, obviamente pero no dependíamos de estos proyectos. Ahora sí" [Hombre, 42 con pareja, sin hijos, segunda postdoc, centro mixto].

Las políticas de austeridad suscitan una gran presión entre el personal de investigación puesto que les exige resultados cuantitativos, de acuerdo a la "excelencia" y meritocracia, mientras también se agudizan las situaciones de precariedad e inestabilidad laboral. En este sentido, un investigador de un centro del área experimental afirma:

"Antes eran contratos de tres, cuatro años, ahora se va reduciendo y, entonces, quieren gente tan especializada que al tercer día ya estén rindiendo. Incluso ahora te ofrecen una postdoc de 10 meses que no tiene ningún sentido porque entre que te acostumbras al llegar y que buscas trabajo para cuando se acabe, ¿cuando trabajas?" [Hombre 34 años, sin pareja ni hijos, postdoc, centro mixto].

La consecución de resultados rápidos a pesar de ser conscientes de que los experimentos requieren tiempo genera frustración y desánimo entre las personas que se dedican a la investigación. En su conjunto, se genera un entorno hostil debido a la alta competitividad que promueve la emergencia de estrategias individualistas (Clarke y Knights, 2015; Morley, 2005). En la siguiente cita, un investigador reflexiona sobre la precariedad y la lógica de la competitividad en la ciencia, cuya consecuencia es una menor atención a la calidad de la investigación:

“¿Cuál es el problema del postdoc? Es que tiene una vida media de dos o tres años, y si quieres hacer una ciencia... experimentos que son muy de impacto, es 
normal, es muy probable que tengas que invertir dos años. ¿Y si no me salen o me salen mal? Que en dos años no sacas nada ¿Quién me va a garantizar una plaza? Esto te motiva pero de otra forma, puede fomentar a largo plazo el hecho que los postdoc se hagan cada vez más individualistas y miren objetivos a medio o corto plazo" [Hombre, 40 años, sin pareja ni hijos, postdoc sénior, centro mixto].

La crisis ha potenciado la situación de precariedad laboral de las personas que se dedican a la investigación, en cualquiera de los centros de investigación incluidos en este estudio. En las universidades con tradición de apoyar las carreras científicas según un modelo meritocrático, se han paralizados las convocatorias de promoción y la de incorporación de nuevas candidaturas. En los centros de investigación los salarios de las becas y contratos de investigación han sido menos atractivos, por lo que las personas se han encontrado con el dilema de aceptar contratos en España aceptando peor salario o en otros países donde los salarios y las ventajas para investigar son más competitivas. En algunos centros, las personas entrevistadas han manifestado quejas sobre la desigualdad salarial entre el personal de investigación de origen extranjero y local puesto que sospechan que para atraerlos se les ofrece mejores condiciones laborales. El hecho de que los sueldos y las condiciones de trabajo sean negociados individualmente asegura esta sospecha entre todas las personas de los centros de investigación. Ello pone de relieve la tensión existente en la política científica sobre la necesidad de atraer talento del extranjero y la de estimular a las personas que conforman la "cantera" local.

La situación es especialmente delicada para las personas que sostienen un largo período de espera sin conseguir una plaza de promoción que les permita abandonar las condiciones de inestabilidad y les proporcione el reconocimiento que merece su esfuerzo: "Estoy intentando aguantar... porque ya no tengo edad de... no tengo ganas de cambiar...mmm... pero sinceramente el mundo de la ciencia, en este momento, es bastante deprimente porque hay falta de crítica..." [Mujer, 49 años, casada segundas nupcias sin hijos, centro mixto]. Para ellas no es sólo una cuestión de justicia meritocrática sino de poder realizar un trabajo de mayor calidad con una menor presión sobre el tiempo de trabajo (Lyon y Woodward, 2004). Poner la ciencia en el centro del trabajo de investigación en vez de la metrificación de los resultados científicos.

Por último, queremos enfatizar el tema de la brecha de género en las posiciones de mayor responsabilidad de la escala profesional, puesto que la ausencia de plazas de promoción por razones económicas ha impedido avanzar en su eliminación o, al menos, la disminución de posiciones disponibles. Como no ha habido una oferta de plazas para estas figuras contractuales, las mujeres han tenido muy pocas oportunidades de alcanzar las categorías de catedrática, profesora de investigación o líder de grupo. Por tanto, los hombres siguen siendo mayoritarios en estas posiciones laborales. Esta investigadora del área de la informática lo expresa así:

“...Entonces, es gente que ha tenido una carrera profesional que empezó en su momento, y que consiguió su puesto, y sigue en él. Entonces, como no hay vacantes... isiguen los que estaban! Y en ese momento eran casi todos hombres..." [Mujer, años, sin pareja ni hijos, centro tecnológico de innovación]. 


\section{CONCLUSIONES: PONER LA VIDA Y LA CIENCIA EN EL CENTRO DE NUESTRA ACTIVIDAD}

El establecimiento de un modelo de carrera científica sirve como garantía de estabilidad, promoción y reconocimiento de los méritos científicos. Sin embargo, tanto la crisis como las prácticas neo-gerencialistas han modificado de manera sustancial las trayectorias profesionales. Por una parte, la falta de recursos económicos ha disminuido las oportunidades laborales de hombres y mujeres en la investigación: disminuyendo el número de ofertas laborales, precarizando las condiciones laborales y los salarios, aumentando la inestabilidad laboral y sosteniendo el techo de cristal de las mujeres investigadoras. Por otra parte, la aplicación de un modelo de excelencia ligado a las prácticas neo-gerencialistas de gestión de los recursos humanos sostiene una dinámica donde se premia la rapidez y cantidad de los resultados científicos en vez de la calidad del trabajo y la armonización con los tiempos requeridos para lograr hallazgos significativos en ciencia. La exigencia es tan alta que los y las investigadoras expresan disconformidad respecto a esta lógica extrema de competitividad, que ha convertido las organizaciones científicas en un ambiente hostil para el conocimiento científico. La rapidez y metrización de los resultados de investigación están generando crecientes críticas sobre el modelo de producción científica que, necesariamente, perjudica a la calidad de los resultados y las condiciones de trabajo en los que se producen.

En este artículo también hemos constatado la diversidad de estrategias de progresión científica entre hombres y mujeres, debido tanto a razones objetivas como subjetivas, donde las razones personales y profesionales se entrecruzan constantemente, dificultando la identificación de una motivación prioritaria por la cual las trayectorias se ralentizan o interrumpen. Las lógicas de cada cultura científica, marcadas tanto por la disciplina como por la institución o instituciones de socialización, definen los retos a los que se enfrentarán y las estrategias de promoción (con una carrera más o menos internacionalizada de sus trayectorias, desarrollada en un solo centro o sin fronteras entre países y laboratorios, etc.). Al esfuerzo personal y la pasión por el trabajo, imprescindibles para desarrollar una carrera en ciencia, hay que añadir la influencia de las redes de apoyo que ejercen una influencia positiva o negativa en sus trayectorias. La aplicación objetiva de los méritos de investigación se presenta como una garantía para asegurar la progresión basada en la meritocracia pero, en general, el conocimiento del entorno y la ayuda de los compañeros y superiores favorecen la consecución de una trayectoria profesional exitosa.

Por una parte, hombres y mujeres investigadores son afectados desigualmente por procesos de valoración de sus resultados, puesto que se reconocen más difícilmente los méritos de las mujeres, se prescinde de ellas en los equipos de trabajo sin razones aparentes o se les encarga tareas menos competitivas (administrativas más que de dirección de la investigación). Estructuras de género organizan las vidas personales de hombres y mujeres, dejando una mayor responsabilidad a las mujeres. Sin embargo, éstas son obligadas a seguir las lógicas y prácticas institucionales que aseguran la progresión de modelos ideales masculinos. Aunque la familia es un factor que incide tanto en las carreras de los hombres como de las mujeres, éstas últimas están marcadas 
por el rol de género adjudicado socialmente, que influye tanto en sus propias decisiones como en las decisiones que se toman acerca de ellas. De este modo, algunas mujeres se quejan de la falta de atención que merece su perfil de investigación para las personas que dirigen los equipos, quienes las relegan a papeles relacionados con la burocracia y la organización del centro. Así, no disponen del estímulo y el tiempo necesario para dedicarse a la creación científica, que impulsará sus carreras profesionales. Es posible que este sentimiento de frustración sea el resultado de una mayor concienciación de las mujeres frente a las dificultades con las que se enfrentan. Es una dinámica que viene de lejos, fruto de la dinámica de subordinación que las mujeres sufren sistemáticamente al incorporarse en los contextos profesionales masculinos.

En definitiva, las personas dedicadas a la investigación viven las tensiones del modelo neo-gerencialista y basado en criterios de excelencia de manera paradójica. Por una parte, se les presiona para generar un mayor número de resultados científicos en un período de tiempo más breve, por otra parte, el ambiente se vuelve más hostil por la elevada competitividad y se precariza su situación laboral. Es más, la inestabilidad laboral se instituye como modelo de progresión en los centros de excelencia y en las universidades al no estar aseguradas la financiación económica de sus plazas de promoción. La movilidad se vuelve una exigencia para el desarrollo de una trayectoria excelente, pero en muchas disciplinas esta dinámica no tiene fin debido a la alta especialización tecnológica y a su modelo de excelencia. La falta de recursos y plazas también extiende esta dinámica a otras áreas, fenómeno que corre en paralelo con la "fuga de cerebros" debido a la crisis. Todos estos factores afectan seriamente a los proyectos vitales y personales, a veces poniendo en una disyuntiva la carrera o los cuidados en sus vidas personales.

Desde el momento en que las mujeres son quienes tienen que demostrar que la vida personal no es un impedimento para lograr los mismos hitos profesionales, son ellas las que deben esforzarse más, demostrar en mayor medida su determinación. Dicho esfuerzo no se reconoce suficientemente, ya que no se valora lo personal como parte de la vida profesional. La separación entre estos dos ámbitos se realiza desde un paradigma de neutralidad que mantiene a las mujeres en una situación de inferioridad. No es que la movilidad internacional sea un impedimento para que las mujeres desarrollen trayectorias científicas excelentes, es que se omite sistemáticamente el hecho de que han tenido que realizar un mayor esfuerzo. Las agencias de evaluación, por ejemplo, justifican que las evaluaciones son totalmente objetivas razonando que los miembros de las comisiones de evaluaciones no deben tener en cuenta incidencias personales que afecten a las trayectorias profesionales de las personas evaluadas. Por tanto, la objetividad consiste en ignorar a las personas que, justamente, son el objeto mismo de la evaluación.

Se vuelve indispensable volcar nuestra atención sobre los derechos laborales del personal investigador, así como en los factores que agudizan la brecha de género, puesto que el contexto de crisis agudiza las dificultades que las mujeres encuentran para incorporarse en figuras de estabilidad científica y posiciones de responsabilidad. A pesar de que encontramos disconformidad con el sistema científico actual, sobre la 
dinámica neo-gerencialista y de excelencia, estas lógicas se aceptan sin casi cuestionamientos. En este punto es importante reivindicar la situación precaria y desigual del personal de investigación para generar espacios colectivos de resistencia y transformación. La entrada de mujeres a posiciones estables y en puestos de responsabilidad es más difícil por el estrangulamiento del número de plazas. Socialmente, las mujeres son consideradas menos resistentes en posiciones de presión que los hombres. En consecuencia, se actúa prefiriendo a los candidatos masculinos puesto que tradicionalmente sólo los hombres deben mantenerse en el mercado laboral, especialmente cuando el mercado laboral es precario. Si las mujeres se retiran no es un problema socialmente preocupante ni un defecto del mercado laboral, por el contrario, se considera que es el resultado de la voluntad de las mujeres o de su quiebra ante las dificultades. Este discurso se repite sin cesar en nuestro imaginario colectivo construido con diferentes retóricas por hombres que imaginan a las mujeres llevando una pesada carga y de las propias mujeres que, desde una posición de seguridad profesional, consideran débiles a las que abandonan.

\section{REFERENCIAS BIBLIOGRÁFICAS}

ASKABIDE (2008): Perfil de clientes de prostitución en Bizkaia. Bilbao: Mensajero.

ACKERS, LOUISE (2004): Managing Work and Family Life in Peripatetic Careers: The Experiences of Mobile Women Scientists in the European Union. Women's Studies International Forum, 27(3), 189-201. doi: 10.1016/j.wsif.2004.03.001

ARTHUR, MICHAEL y DENISSE ROSSEAU (1996): The Boundaryless career: A New Employment Principle for a New Organizational Era. New York: Oxford University Press.

BAGILHOLE, BARBARA y JACKIE GOODE (2001): The Contradiction of the Myth of Individual Merit, and the Reality of a Patriarchal Support System in Academic Careers: A Feminist Investigation. European Journal of Women's Studies, 8(2), 161-180. doi: 10.1177/135050680100800203

BAKER, MAUREEN (2010). Career confidence and gendered expectations of academic promotion. Journal of Sociology, 46(3), 317-334. doi: $10.1177 / 1440783310371402$

BOZEMAN, BARRY, DANIEL FAY y CATHERINE P. SLADE (2013): Research collaboration in universities and academic entrepreneurship: the-state-of-the-art. The Journal of Technology Transfer, 38(1): 1-67. doi: 10.1007/s10961-012-92818

BROCKLEHURST, MICHAEL (2003): Self and Place: A Critique of the 'Boundaryless Career', CMS3, https://goo.gl/48bV6N 1/05/2016. 
CLARK, BURTON, R. (1998): Creating Entrepreneurial Universities: organisational pathways of transformation. New York: Elsevier.

CLARKE, CAROLINE A. y DAVID KNIGHTS (2015): Careering through academia: Securing identities or engaging ethical subjectivities? Human Relations, 68 (12), 1865-1888. doi: 10.1177/0018726715570978

DEEM, ROSEMARY (1998): 'New managerialism' and higher education:

The management of performances and cultures in universities in the United Kingdom. International Studies in Sociology of Education, 8(1), 47-70. doi: 10.1080/0962021980020014

DEEM, ROSEMARY (2001): Globalisation, New Managerialism, Academic Capitalism and Entrepreneurialism in Universities: is the local dimension still important? Comparative $\quad$ Education, 37(1), http://www.jstor.org/stable/3099730

EUROPEAN COMMISSION (2005): The European Charter for Researchers. The Code of Conduct for the Recruitment of Researchers. Brussels: DirectorateGeneral for Research.

FASSA, FARINAZ (2013): La recherche en Suisse quand le genre interroge les critères de l'excellence scientifique, Mouvements 4, 76, 162-175.

FUSULIER, BERNARD (2016): L'expérience de la carrière scientifique aujourd'hui. Zoom sur le vécu de chercheurs postdoctorants, M/S: médecine sciences, 32, 3, 297-302. doi:10.1051/medsci/20163203015

GONZÁLEZ, ANA MARÍA y VERGÉS, NÚRIA (2013): Beyond the Work-Life Balance: Family and International Mobility of the Highly Skilled, Sociología y Tecnociencia. Revista Digital de Sociología del Sistema Tecnocientífico, 3: 55-76

GONZÁLEZ, ANA MARÍA (2014) "Inclusion of Women in Science. Long-term strategies for alone or with partners' women", Géneros. Multidisciplinary Journal of Gender Studies, 3 (3): 459-482.

GONZÁLEZ, ANA MARÍA y TORRADO, ESTHER. (2014): "Familia e Investigación: Estrategias familiares y de género de las investigadoras españolas", Revista Feminismo/s, 23: 183-205

GONZÁLEZ, ANA MARÍA, NAVARRETE, JOSÉ, Y CABRERA, ESTHER (2015): Dancers in the Dark: Scientific Careers According to a Gender-Blind Model of Promotion. Interdisciplinary Science Reviews, 40(2): 182-202. 
INKSON, KERR, HUGH GUNZ, SHIV GANESH y ROPER, JULIA (2012): Boundaryless Careers: Bringing Back Boundaries. Organization Studies, 33(3), 323-340. doi: 10.1177/0170840611435600

KELAN, ELISABETH K. (2009): Gender Fatigue: The Ideological Dilemma of Gender Neutrality and Discrimination. Organisations, Canadian Journal of Administrative Sciences, 26, 197-210. doi: 10.1002/cjas.106

LYON, DAWN y ALISON E. WOODWARD (2004): Gender and Time at the Top: Cultural Construction of Time in High-Level Careers and Homes. European Journal of Women's Studies, 11(2), 205-221. doi: 10.1177/1350506804042096

MORLEY, LOUISE (2005): The Micropolitics of Quality. Critical Quarterly, 47(1-2), 83-95. doi: 10.1111/j.0011-1562.2005.00616.x

MORLEY, LOUISE (2016). Troubling intra-actions: gender, neo-liberalism and research in the global academy. Journal of Education Policy, 31 (1), 28-45. doi: 10.1080/02680939.2015.1062919

WINNIFRED (2015): For Slow Scholarship: A Feminist Politics of Resistance through Collective Action in the Neoliberal University. ACME, International E-Journal for Cultural Geographies, 14(4), $1235-1259$. doi : 10.1080/00131857.2014.880645

REES, TERESA (2011): The Gendered Construction of Scientific Excellence. Interdisciplinary Science Reviews, 36(2), 133-145. doi: 10.1179/030801811X13013181961437

ROPER, JULIET, SHIV GANESH y KERR INKSON (2010): Neoliberalism and knowledge interests in boundaryless careers discourse. Work, Employment and Society, 24, 661-679. doi: 10.1177/0950017010380630

SCULLY, MAUREEN A. (2002). Confronting Errors in the Meritocracy. Organization, 9(3), 396-401. doi: 10.1177/135050840293004

SLAUGHTER, SHEILA y GARY RHOADES (2000). The neo-liberal university. New Labor Forum, (6), 73.

SLAUGHTER, SHEILA y GARY RHOADES (2004): Academic capitalism and the new economy: Markets, state, and higher education. Baltimore: Johns Hopkins University Press.

SULLIVAN, SHERRY E. y MICHAEL ARTHUR (2006): The Evolution of the Boundaryless Career Concept: Examining Physical and Psychological Mobility. Journal of Vocational Behaviour, 69, 19-29. doi: 10.1016/j.jvb.2005.09.001 
TAYLOR, STEVE J. y ROBERT BODGAN (1984): Introducción a los métodos cualitativos en investigación. La búsqueda de los significados. España: Paidós.

TROW, MARTIN y PAUL CLARK (1994): Managerialism and the Academic Profession: Quality and Control. Higher Education Report No. 2. Open University. Quality Support Centre. doi: 10.2753/RES1060-9393540304

VAN DEN BRINK, MARIEKE y YVONNE BENSCHOP (2012): Gender practices in the construction of academic excellence: Sheep with five legs. Organization, 19, 507-524. doi: 10.1177/1350508411414293

VÁZQUEZ-CUPEIRO, SUSANA y MARY ANN ELSTON (2006): Gender and academic career trajectories in Spain: From gendered passion to consecration in a Sistema Endogámico? Employee Relations, 28(6), 588-603. doi: $10.1108 / 01425450610704515$

XIE, YIE (2010): Gender asymmetry in family migration: Occupational inequality or interspousal comparative advantage? Journal of Marriage and Family, 72, 37592. doi: $10.1111 / \mathrm{j} .1741-3737.2010 .00706 . x$

YEOH, BRENDA S. A. y LOUISA-MAY KHOO (1998): Home, Work and Community: Skilled Migration and Expatriate Women in Singapore. International Migration, 36(2), 159-186. doi: 10.1111/1468-2435.00041 